\title{
ASPECTOS DE QUALIDADE E SEGURANÇA DO TAMBAQUI (COLOSSOMA MACROPOMUM) E PINTADO DA AMAZÔNIA (PSEUDOPLATYSTOMA RETICULATUM X LEIARIUS MARMORATUS)
}

Quality and safety aspects of the tambaqui (Colossoma macropomum) and painted from amazon (Pseudoplatystoma reticulatum $x$ Leiarius marmoratus)

Aspectos de calidad y seguridad de la cachama (Colossoma macropomum) y pintado de amazon (Pseudoplatystoma reticulatum $x$ Leiarius marmoratus)

Maria Olivia dos Santos Oliveira*1,2, Danielle de Bem Luiz ${ }^{4}$, Viviane Rodrigues Verdolin dos Santos ${ }^{3}$, Eduardo Henrique Silva de Oliveira ${ }^{2}$, Glêndara Aparecida de Souza Martins ${ }^{1,2}$ ${ }^{1}$ Programa de Pós-Graduação em Ciência e Tecnologia de Alimentos, Universidade Federal do Tocantins, Palmas TO, Brasil.

${ }^{2}$ Laboratório de Cinética e Modelagem de Processos, Universidade Federal do Tocantins, Palmas - TO, Brasil.

${ }^{3}$ Embrapa Pesca e Aquicultura, Palmas - TO, Brasil.

${ }^{4}$ Embrapa Agropecuária Oeste, Dourados - MS, Brasil.

* Correspondência: Universidade Federal do Tocantins (UFT), Câmpus de Palmas, Avenida NS 15, Quadra 109 Nortel Plano Diretor Norte Bloco II, sala 35B, Palmas/TO, CEP: 77001-090.e-mail: mariaoliviaeng@gmail.com

Artigo recebido em 29/04/2019 aprovado em 03/05/2019 publicado em 16/06/2019.

\section{RESUMO}

Este trabalho tem como objetivo apresentar uma revisão bibliográfica à cerca de fatores que influenciam na qualidade e segurança de duas espécies nativas de pescado da produção ao processamento, o tambaqui e o surubim. Como o pescado é um alimento altamente perecível, é necessário que haja cuidados durante seu trajeto até o consumidor final e para isso é importante que se conheça a espécie trabalhada e fatores que poderão influenciar na qualidade e segurança da mesma em relação ao consumidor.

Palavras-chave: tambaqui, qualidade, processamento.

\section{ABSTRACT}

This work aims to present a literature review about the factors that influence the quality and safety of two native fish species from production to processing, tambaqui and surubim. As fish is a highly perishable food, it is necessary to take care during its journey to the final consumer and for this it is important to know the species worked and factors that may influence the quality and safety of the same in relation to the consumer.

Keywords: tambaqui, quality, processing.

\section{RESUMEN}

Este trabajo tiene como objetivo presentar una revisión bibliográfica a cerca de factores que influencian en la calidad y seguridad de dos especies nativas de pescado de la producción al procesamiento, el tambaqui y el surubim. Como el pescado es un alimento altamente perecedero, es necesario que haya cuidados durante su trayecto hasta el consumidor final y para ello es importante que se conozca la especie trabajada y factores que podrán influir en la calidad y seguridad de la misma en relación al consumidor.

Descriptores: Tambaqui, calidad, elaboración. 


\section{INTRODUÇÃO}

O consumo mundial de pescado vem apresentando grande crescimento nos últimos anos, chegando a 20,3 kg per capita em 2016, um aumento de $28 \%$ no consumo quando comparado aos $14,4 \mathrm{~kg}$ consumido em 1990. Isto graças ao setor da aquicultura que passou a fornecer mais da metade do pescado para consumo humano. Espera-se que o Brasil até 2025 apresente um aumento de 104\% na aquicultura e produção de pescado, superando o México com 54,2\% e a Argentina com 53,9\% (FAO, 2018). A aquicultura no Brasil tem sido promissora devido a fatores como a abundância de recursos naturais. O país concentra cerca de $14 \%$ de toda água doce disponível no planeta, o clima é predominante tropical e apresenta grande produção de grãos utilizados para fabricação de ração para alimentação de pescado (Tundisi \& Tundisi, 2016).

Entre as espécies mais consumidas, processadas em indústrias e que apresentam grande potencial para cultivo no Brasil, estão os tambaquis e surubins. O tambaqui (Colossoma macropomum) é o segundo maior peixe de escamas da bacia amazônica, e a espécie nativa mais cultivada do país (Pedroza Filho, 2016). Já o pintado da Amazônia ou surubim híbrido (Pseudoplatystoma reticulatum X Leiarius marmoratus) apresenta maior potencial de rendimento cárneo que as espécies puras (Oliveira et al., 2014), tornando sua carne altamente apreciada.

A qualidade e segurança do pescado dependem das condições e cuidados desde a criação da matéria-prima até o produto que chega à mesa do consumidor (Argenta, 2012). Dentre os contaminantes de ordem microbiológica perigosos e frequentes estão a Salmonella sp., Staphylococcus coagulase positiva e coliformes termotolerantes, no grupo de coliformes o patógeno Escherichia coli (Pinheiro, 2015).
Além disso, um fator importante é a composição centesimal do peixe, que pode variar entre uma espécie e outra, devido a vários fatores, entre eles estão alimentação e cultivo. Por isso é necessário o conhecimento da composição da espécie durante determinadas épocas de engorda e condição de cultivo (Falch et al., 2010).

\section{REVISÃO DE LITERATURA}

\section{Principais espécies nativas}

Entre as principais espécies consumidas e processadas no Brasil, estão os tambaquis e surubins. O tambaqui, Colossoma macropomum, é o maior da ordem Characiforme da Bacia Amazônica (Lima, 2016) e está largamente distribuído na América do Sul. $\mathrm{Na}$ natureza, pode alcançar cerca de 1 metro de comprimento e até $30 \mathrm{~kg}$ de peso sendo considerado o segundo maior peixe de escamas da bacia amazônica, perdendo em porte somente para o pirarucu (Arapaima gigas). Apresenta grande capacidade de metabolizar proteína animal e vegetal, podendo ser onívoro com tendência a herbívoro, filtrador e frugívoro, ou seja, se adapta facilmente a alimentação fornecida (LoperaBarrero, 2011).

O tambaqui apresenta características morfológicas e fisiológicas diferentes de acordo com sua origem. Tambaquis de cultivo geralmente são mais arredondados, apresentam nadadeiras um pouco mais longas e poucas imperfeições, além de mais corados. Já os tambaquis capturados nos rios têm corpo mais alongado, sinais de esfolamento e nadadeiras com mais imperfeições (mordidas, rasgos etc). Geralmente é percebida a diferença no sabor de ambos, uma vez que a produção de cativeiro é associada a "gosto de barro" e a de rios tem um sabor mais suave e uma maior qualidade, isso pode ser devido à alimentação, pois em cativeiro os peixes são 
alimentados com ração e em rios se alimentam de frutos (Lima, 2016).

Em 2016, o tambaqui foi a segunda espécie mais cultivada no Brasil com 136,99 mil toneladas, ou $27 \%$ do total nacional (IBGE, 2016). A facilidade de produção e o rápido crescimento tornaram o tambaqui a espécie nativa mais cultivada e mais popular da piscicultura brasileira (Pedroza Filho, 2016). Seu cultivo é possível em todo o Brasil, mas tem se concentrado no Norte, Nordeste e Centro-Oeste, devido sua aceitação no mercado e a baixa mortalidade de alevinos a altas temperaturas, tornando estes lugares propícios a sua criação e comercialização (Oliveira, 2017).

A ordem Siluriforme está inserida no grande grupo dos peixes, suas principais características são à ausência de escamas e pele espessa, tornando-o popularmente chamado de "peixe de couro". Morfologicamente apresenta corpo alongado e cilíndrico, cabeça deprimida e mandíbula mais curta que a maxilar superior e dentes viliformes no palato (Santos, 2017). Na ordem Siluriformes, está a família Pimelodidae, onde estão inseridos o jundiá amazônico (Leiarius marmoratus), surubins e cacharas, representantes do gênero Pseudoplatystoma (Gomides, 2011). Tornando possível o cruzamento entre a femêa do cachara (Pseudoplatystoma reticulatum) e o macho do jundiá (Leiarius marmoratus), originando um híbrido, Pintado da Amazônia (Marques, 2016).

O jundiá é uma espécie amazônica, em seu ambiente natural, apresenta hábito alimentar piscívoro, mas, demonstram tendências onívoras quando cultivado no fundo dos rios, onde a temperatura da água varia de 24 a $26^{\circ} \mathrm{C}$ (RamírezMerlano et al., 2010). Geralmente incorporado a piscicultura devido a apreciação de sua carne, e suas características siluriformes, que não apresentam espinhos intramusculares em "y" e ausência de escamas (Honorato, 2015).

Já o cachara é nativo das bacias dos rios Paraná e do Médio Amazonas. Pode ser caracterizado por suas linhas escuras verticais, conectando-se formando células na região dorsal e lateral, na cabeça e cauda apresenta linhas ou manchas também escuras (Buitrago-Suárez, 2007).

Os híbridos de surubim apresentam maior percentagem de corpo, indicando maior potencial de rendimento cárneo que as espécies puras (Oliveira et al., 2014). Em 2016, a produção de surubins híbridos e não-híbridos, ocupou o $5^{\circ}$ lugar no Brasil, com um total de 20,437 mil toneladas despescadas, 4,3\% da produção nacional de todas as espécies produzidas (IBGE, 2016).

\section{Padrão de identidade e qualidade e legislação nacional}

O Regulamento de Inspeção Industrial e Sanitária de Produtos de Origem Animal (RIISPOA) prevê normas que regulam em todo território nacional, a inspeção industrial e sanitária de produtos de origem animal. Ele trata o pescado como denominação genérica, abordando características que o pescado fresco e seus derivados devem apresentar para que sejam próprios para o consumo (Brasil, 1952). Além do RIISPOA, existem os Regulamentos Técnicos de Identidade e Qualidade (RTIQs), que apresentam condições mínimas exigíveis para elaboração e embalagem de pescados e derivados (Brasil, 1997).

Entende-se por peixe fresco, o produto obtido de espécimes saudáveis e de qualidade adequada ao consumo humano, convenientemente lavado e que seja conservado somente pelo resfriamento a uma temperatura próxima a do ponto de fusão do gelo (Brasil, 1997).

O RIISPOA, no artigo $\mathrm{n}^{\circ} 438$, denomina genericamente "PESCADO" como peixes, crustáceos, 
moluscos, anfíbios, quelônios e mamíferos de água doce ou salgada, usados na alimentação humana. Classifica o pescado em natureza como fresco, resfriado e congelado. Onde "fresco" é o pescado que somente teve gelo adicionado, sem sofrer qualquer outro tipo de processo de conservação. Entende-se por "resfriado" o pescado mantido em temperatura entre 0,5 e $-2^{\circ} \mathrm{C}$, devidamente acondicionado em gelo. Pescado "congelado", o tratado por processos adequados de congelação, em temperatura não superior a $-25^{\circ}$ C. De acordo com o artigo $n^{\circ} 444$, o julgamento das condições sanitárias do pescado resfriado e do congelado é realizado de acordo com as normas previstas para o pescado fresco, naquilo que lhes for aplicável (Brasil, 1952).

Para o Regulamento Técnico de Identidade e Qualidade de Peixe Fresco (Inteiro e Eviscerado) os peixes podem ser denominados como animais aquáticos de sangue frio, excluindo assim, os mamíferos aquáticos, os animais invertebrados e os anfíbios. Denomina também peixe fresco, como produto obtido de espécimes saudáveis e de qualidade adequada ao consumo humano, convenientemente lavado e que seja conservado somente pelo resfriamento a uma temperatura próximo a do ponto de fusão do gelo. Podendo ser classificado em inteiro (peixe inteiro somente lavado) e eviscerado (peixe fresco que teve suas vísceras retiradas) podem ser apresentados com ou sem cabeça, nadadeira ou escamas (Brasil, 1997).

Para que o peixe fresco (inteiro ou eviscerado) seja próprio para o consumo deve apresentar sensorialmente algumas características. $\mathrm{O}$ produto deverá apresentar-se com todo o frescor da matéria prima convenientemente conservada, deverá estar isento de qualquer evidência de decomposição, manchas por hematomas, coloração distinta à normal para a espécie considerada, incisões ou rupturas das superfícies externas. As escamas devem estar unidas entre si e fortemente aderidas à pele, devendo ser translúcidas, com brilho metálico e não devem ser viscosas. A pele deve estar úmida, tensa e bem aderida. As espécies que possuem mucosidade devem apresenta-la, na forma aquosa e transparente. Os olhos devem ocupar a cavidade orbitária e ser brilhantes e salientes. O opérculo deve oferecer resistência à sua abertura, sua face interna deve ser nacarada, os vasos sanguíneos cheios e fixos. As brânquias devem estar da cor rosa ao vermelho intenso, úmidas e brilhantes, ausência ou discreta presença de muco. O Abdome tenso, sem diferença externa com a linha ventral, peritônio deverá apresentar-se muito bem aderido às paredes, as vísceras inteiras, bem diferenciadas, brilhantes e sem dano aparente. Os músculos devem estar aderidos aos ossos fortemente e de elasticidade marcante. Odor, cor e sabor, devem apresentar característicos à espécie que se trate (Brasil, 1997).

$\mathrm{O}$ artigo $\mathrm{n}^{\circ} 445$ do RIISPOA, estabelece características que condenam o pescado como impróprio para o consumo. Este não deve apresentar aspecto repugnante, mutilado, traumatizado ou deformado. Assim como coloração, cheiro ou sabor anormais. Não portar lesões ou doenças microbianas que possam prejudicar a saúde do consumidor. Ausência de infestação muscular maciça por parasitas, que possam prejudicar ou não a saúde do consumidor. Que não tenha sido tratado por antissépticos ou conservadores não aprovados pelo Departamento de Inspeção de Produtos de Origem Animal (DIPOA). Não ser provenientes de água contaminadas ou poluídas. Não tenha procedente de pesca realizada em desacordo com a legislação vigente ou recolhido já morto, salvo quando capturado em operações de pesca. Pescado em mau estado de conservação e que não se enquadre nos limites físicos e químicos fixados para o pescado fresco (Brasil, 1952). 


\section{Indicadores físico-químicos e microbiológicos de qualidade}

A legislação para pescado "in natura" ou fresco não estabelece limites para composição centesimal, pois os mesmos podem variar, entre uma espécie e outra, e dentro de uma mesma espécie, de acordo com a época do ano, do tipo e quantidade de alimento disponível, da qualidade da dieta consumida, do estágio de maturação sexual, da idade, das condições de cultivo e da parte do corpo analisada (Viana, 2013; Aguiar et al., 2018)

O RIISPOA determina os seguintes limites físicos e químicos para caracterização do pescado fresco: reação negativa de gás sulfídrico e de indol, nos quais o limite e máximo de indol será de 4 gramas por 100 gramas; $\mathrm{pH}$ de carne externa inferior a 6,8 e da interna, inferior a 6,5 nos peixes; bases voláteis total inferiores a 30 centigramas de nitrogênio (processo de difusão) por 100 g de carnes; bases voláteis terciárias inferiores a quatro miligramas por cento de nitrogênio em $100 \mathrm{~g}$ de carne (Brasil, 1952).

No Regulamento Técnico de identidade e qualidade do peixe fresco inteiro ou eviscerado, são exigidos: bases voláteis totais: inferior a 30mg de nitrogênio por 100 gramas de carne, excluído os Elasmobrânquios; nível máximo de histamina de 100ppm no músculo, nas espécies pertencentes às famílias Scombridae, Scombresocidae, Clupeidae, Coryyphaenidae (Brasil, 1997).

A composição do pescado pode variar entre 60 e $85 \%$ de umidade, aproximadamente $20 \%$ de proteína bruta, 1 a $2 \%$ de cinzas e 0,6 a $36 \%$ de lipídios (Minozzo, 2011).

A Tabela de Composição de Alimentos da Amazônia descreve algumas espécies com sua determinada composição corporal, para tambaqui inteiro cru, os valores são $72,70 \%$ para umidade, $19 \%$ proteína, 1,4\% de cinzas e 6,9\% de lipídeos (Aguiar, 1996). Na tabela de Composição de Alimentos
(TACO) é possível encontrar alguns valores médios para algumas espécies, para pintado cru (partes comestíveis) apresenta 80,3\% de umidade, $18,3 \%$ de proteína, $1,1 \%$ de cinzas e $1,3 \%$ de lipídios (UNICAMP, 2011). A composição da sardinha inteira crua pode ser encontrada nas duas tabelas, com umidades respectivamente de $58,5 \%$ e $76,6 \%$, e teores de proteína de 18,5\% e 21,1\% (Aguiar, 1996; UNICAMP, 2011). Comprovando assim que a composição varia de acordo com vários fatores extrínsecos e intrínsecos.

A água é um dos componentes do peixe que apresenta maiores variações relacionadas às espécies e às épocas do ano, podendo compreender de 53 a $80 \%$ do total. Como é o principal componente, sua presença ou ausência pode influenciar na qualidade na coloração, suculência, textura e sabor da carne (Minozzo, 2016)

A maioria dos componentes nitrogenados do pescado faz parte das proteínas. O conhecimento da composição e das propriedades dos diversos componentes nitrogenados é de grande relevância prática, uma vez que as características próprias do músculo dependem, em grande parte, da concentração e da proporção desses componentes (Argenta, 2012).

Já o conteúdo de gordura do pescado sofre variações muito significativas, dependendo da época do ano, da dieta, da temperatura da água, da salinidade, da espécie, do sexo e da parte do corpo analisada (Minozzo, 2011)

No entanto, o pescado não estará adequado para o consumo se estiver fora dos padrões microbiológicos, estes que estão relacionados com sua qualidade e segurança. Os procedimentos de higienização são essenciais para garantir a qualidade e segurança dos produtos. Assim, a utilização de cuidados rigorosos de higiene, seguindo normas adequadas, favorece o controle da qualidade, viabiliza os custos de produção, satisfaz os consumidores, e 
ainda respeita as normas e padrões microbiológicos recomendados pela legislação vigente (Junior, 2016).

A Agência Nacional de Vigilância Sanitária (ANVISA) estabelece pela Resolução - RDC n ${ }^{\circ} 12$, de 2 de janeiro de 2001, padrões microbiológicos e sanitários para alimentos, com critérios para interpretação e conclusão de análises. Para as amostras indicativas de pescado, ovas de peixes, crustáceos e moluscos cefalópodes "in natura", resfriados ou congelados não consumidos crus, as tolerâncias para Staphylococcus coagulase positiva e Salmonella sp são de $10^{3}$ microorganismos/g e ausência em $25 \mathrm{~g}$, respectivamente (Brasil, 2001). Na legislação da ANVISA não há limite definido para Coliformes ou E. coli para a categoria de pescado fresco ou "in natura".

Costa el al. (2016) identificaram $95 \%$ de contaminação microbiológica em pescados comercializados no interior de São Paulo. O pescado, principalmente fresco está no topo da lista de alimentos associado à contaminação microbiológica, devido a sua vulnerabilidade, consequência de sua composição, manipulação, transporte, acondicionamento e de como é comercializado (Junior, 2016).

\section{CONCLUSÃO}

Embora o consumo de pescados no Brasil ainda seja baixo, essa é a carne mais demandada mundialmente e, tanto no país quanto no mundo, seu consumo vem crescendo de forma acelerada. Os peixes quando não manipulados ou armazenados adequadamente podem sofrer alterações físicoquímicas e microbiológicas, culminando na perca de qualidade. Portanto os parâmetros apresentados tornam-se necessários para que o produto final chegue seguro à mesa do consumidor.

Todos os autores declararam não haver qualquer potencial conflito de interesses referente a este artigo.

\section{REFERÊNCIAS}

AGUIAR, G.P.S. MARTINS, V.G. MARTINS, P.C.C. BOSCHERO, R.A. PRENTICEHERNÁNDEZ, C. Produção de lipase microbiana a partir de resíduos de corvina. Revista de Engenharia e Tecnologia, v. 10, n. 1, p. 118-129, 2018.

AGUIAR, J.P.L. Tabela de composição de alimentos da Amazônia, Acta Amazônica, v.26, n.1-2, p.121126, 1996.

ARGENTA, F.F. Tecnologia de Pescado: Características e Processamento da Matéria Prima. Porto Alegre, RS. Trabalho de conclusão de curso. Universidade Federal do Rio Grande do Sul - UFRS; 2012.

BRASIL. Ministério da Saúde. Secretaria de Vigilância Sanitária (SVS). 1997. Regulamento técnico sobre as condições higiênico-sanitárias e de boas práticas de fabricação para estabelecimentos produtores e industrializadores de alimentos. Diário Oficial [da] República Federativa do Brasil, BrasíliaDF, 01 de agosto.

BRASIL. Ministério da Agricultura, Pecuária e Abastecimento. 1952. Regulamento da Inspeção Industrial e Sanitária dos Produtos de Origem Animal (RIISPOA). 154 p.

BRASIL. Ministério da Agricultura, Pecuária e Abastecimento. Portaria $n^{\circ} 185$ de 1997. Aprova o regulamento técnico de identidade e qualidade de peixe fresco (inteiro e eviscerado). Diário Oficial da República Federativa do Brasil, Brasília - DF, 13 maio.

BRASIL. Agência Nacional de Vigilância Sanitária do Ministério da Saúde. Resolução ${ }^{\circ}$ 12, 2001. Aprova o Regulamento Técnico Sobre Padrões Microbiológicos para Alimentos. Diário Oficial [da] União, BrasíliaDF, 02 de janeiro.

BUITRAGO-SUAREZ, U.A. BURR, B.M. Taxonomy of the catfish genus Pseudoplatystoma Bleeker (Siluriformes: Pimelodidae) with recognition of eight species. Zootaxa, v. 1512, n. 1, p. 1-38, 2007.

FALCH, E. OVERREIN, I. SOLBERG, C. SLIZYTE, R. Composition and Calories. In: NOLLET, L.M.L; TOLDRÁ, F. Handbook of Seafood and Seafood Products Analysis. Boca Raton, FL: CRC Press Taylor \& Francis Group, p. 257 - 285, 2010.

FAO. The State of World Fisheries and Aquaculture 2018. Contributing to food security and nutrition for all. Rome. 227 pp. 
GOMIDES, P.F.V. Densidade de estocagem do híbrido pintado amazônico (Pseudoplatystoma tigrinum fêmea $X$ Leiarius marmoratus macho) em viveiros escavados. Goiânia, GO. Dissertação de Mestrado. Universidade Federal de Goiás-UFG; 2011.

HONORATO, C.A. CANEPPELE, A. MATOSO, J.C. PRADO, M.R. SIQUEIRA, M.S. SOUZA, L.R.O. Caracterização física de filés de Surubim (Pseudoplatystoma sp.), Pacu (Piaractus mesopotamicus) e Pirarucu (Arapaimas gigas). Arquivos de Ciências Veterinárias e Zoologia da UNIPAR, v. 17, n. 4, 2015.

JUNIOR, A.C.S.S. BARBOSA, F.H.F. MONTEIRO, J. F. Aspectos higiênico-sanitários na comercialização no mercado de pescado igarapé das mulheres, MacapáAP. Biota Amazônia, v. 6, n. 4, p. 15-19, 2016.

LIMA, M.P. Efeitos das mudanças climáticas sobre a expressão gênica e a fisiologia do tambaqui (Colossoma macropomum, Cuvier, 1818). Manaus, AM. Tese de doutorado. Instituto Nacional de Pesquisas da Amazônia - INPA; 2016.

LOPERA-BARRERO, N.M. RIBEIRO, R.P. POVH, J.A. VARGAS, L.D.M. POVEDA-PARRA, A.R. \& DIGMAYER, M. As principais espécies produzidas no Brasil. Produção de organismos aquáticos: uma visão geral no Brasil e no mundo. Agrolivros. Guaíba, p. 143-215, 2011.

MARQUES, S.A.D. Determinação do coeficiente de digestibilidade aparente de alimentos para pintado amazônico (Pseudoplatystoma reticulatum x Leiarius marmoratus). Sinop, MT. Tese de Doutorado. Universidade Federal de Mato Grosso; 2016.

MINOZZO, M.G. Processamento e Conservação do Pescado. Curitiba: E-tec Brasil, 2011. 166 p.

OLIVEIRA, A.M.S. DE OLIVEIRA, C.A.L. RODRIGUES, R.A. SANCHEZ, M.S.S. NUNES, A.L. FANTINI, L.E. DE CAMPOS, C.M. Crescimento de juvenis de Pseudoplatystoma reticulatum e Pseudoplatystoma spp. em viveiro. Semina: Ciências Agrárias, v. 35, n. 2, p. 1091-1098, 2014.

OLIVEIRA, C.M. SOUSA, R.G.C. Cultivo de tambaquis da pré-engorda ao abate com diferentes taxas de arraçoamento. Biota Amazônia, v. 7, n. 4, p. 20-25, 2017.

PEDROZA FILHO, M.X. RODRIGUES, A.P.O. REZENDE, F.P. Dinâmica da produção de tambaqui e demais peixes redondos no Brasil. Boletim Ativos da AQUICULTURA, EMBRAPA. V. 2, P. 1-5, 2016.
PINHEIRO, V.S. BISCONSIN-JUNIOR, A. CARVALHO, A.L. Coliformes totais e termotolerantes em tambaqui comercializado no mercado municipal de Ariquemes-RO. In: Anais do $5^{\circ}$ simpósio de Segurança Alimentar, Alimentação e Saúde, p. 26-29, 2015.

RAMÍREZ-MERLANO, J.A. OTERO-PATERNINA, A.M. CORREDOR-SANTAMARÍA, W. MEDINAROBLES, V.M. CRUZ-CASALLAS, P.E. VELASCO-SANTAMARÍA. Y.M. Utilización de organismos vivos como primera alimentación de larvas de yaque (Leiarius marmoratus) bajo condiciones de laboratorio. Revista Orinoquia, v. 14, n. 1, p. $45-58,2010$.

SANTOS, M. F. Sistemática filogenética e evolução cromossômica em espécies amazônicas de Aspredinidae (Ostariophysi, Siluriformes). Manaus, AM. Tese de Doutorado. Instituto Nacional de Pesquisas da Amazônia - INPA; 2017.

TUNDISI, J. G.; TUNDISI, T. M. Limnologia. 1 ed., São Paulo, Oficina de textos, 2016.

Universidade Estadual de Campinas. Tabela Brasileira de Composição de Alimentos - TACO. Campinas, 4ª Edição, P. 42-44; 2011.

VIANA, Z.C.V. SILVA, E. FERNANDES, G.B. SANTOS, V.L.C.S. Composição centesimal em músculo de peixes no litoral do estado da Bahia, Brasil. Revista de Ciências Médicas e Biológicas, v. 12, n. 2, p. 157-162, 2013. 